Technical Notes

\title{
Investigation of an SFOV hybrid gamma camera for thyroid imaging
}

\author{
S.L. Bugby ${ }^{\text {a,* }}$, J.E. Lees ${ }^{a}$, A.H. Ng ${ }^{\text {b }}$, M.S. Alqahtani ${ }^{\text {a,c }}$, A.C. Perkins ${ }^{b}$ \\ a Space Research Centre, Department of Physics and Astronomy, University of Leicester, Leicester LE1 7RH, UK \\ ${ }^{b}$ Radiological Sciences, School of Medicine, University of Nottingham, Nottingham NG7 2UH, UK \\ ${ }^{\mathrm{c}}$ Radiological Sciences Department, College of Applied Medical Sciences, King Khalid University, Postcode: 3665, Zip Code: 16481, Abha, Saudi Arabia
}

\section{A R T I C L E I N F O}

\section{Article history:}

Received 15 May 2015

Received in revised form 24 November 2015

Accepted 3 December 2015

Available online 6 January 2016

\section{Keywords:}

SFOV gamma camera

Image contrast

Thyroid scintigraphy

\section{A B S T R A C T}

The Hybrid Compact Gamma Camera (HCGC) is a small field of view (SFOV) portable hybrid gammaoptical camera intended for small organ imaging at the patient bedside. In this study, a thyroid phantom was used to determine the suitability of the HCGC for clinical thyroid imaging through comparison with large field of view (LFOV) system performance.

A direct comparison with LFOV contrast performance showed that the lower sensitivity of the HCGC had a detrimental effect on image quality. Despite this, the contrast of HCGC images exceeded those of the LFOV cameras for some image features particularly when a high-resolution pinhole collimator was used.

A clinical simulation showed that thyroid morphology was visible in a 5 min integrated image acquisition with an expected dependency on the activity within the thyroid. The first clinical use of the HCGC for imaging thyroid uptake of ${ }^{123} \mathrm{I}$ is also presented.

Measurements indicate that the HCGC has promising utility in thyroid imaging, particularly as its small size allows it to be brought into closer proximity with a patient. Future development of the energy response of the HCGC is expected to further improve image detectability.

(C) 2015 Associazione Italiana di Fisica Medica. Published by Elsevier Ltd. This is an open access article under the CC BY license (http://creativecommons.org/licenses/by/4.0/).

\section{Introduction}

The number of small field of view (SFOV) gamma cameras in development or available to clinicians is increasing [1]. These cameras are often designed for specific tasks such as sentinel lymph node biopsy or for small organ imaging. The imaging performance of SFOV cameras may be characterised through test protocols (e.g. [2]) or through phantom simulations of specific clinical situations.

One small organ well-suited for SFOV gamma imaging is the thyroid, a superficial gland positioned in the neck. Thyroid physiology and morphology can both be investigated through gamma imaging, with clinicians looking for regions of unusually high or low radioisotope uptake. Thyroid imaging commonly uses ${ }^{99 \mathrm{~m}} \mathrm{Tc}-$ pertechnetate (for morphology) or ${ }^{123}$ I-sodium iodide (for true metabolic imaging) [3].

In this communication, a thyroid phantom was used to determine the suitability of an SFOV handheld gamma camera for clinical thyroid imaging through comparison with large field of view (LFOV) system performance. An example of the first clinical use for a patient undergoing thyroid imaging is also presented.

\footnotetext{
* Corresponding author. Space Research Centre, Department of Physics and Astronomy, University of Leicester, Leicester LE1 7RH, UK. Tel: +44 116229 7720; fax: +44 1162522464 .

E-mail address: s.bugby@le.ac.uk(S.L. Bugby).
}

\section{Materials and methods}

\section{SFOV camera}

These studies used the Hybrid Compact Gamma Camera (HCGC), an SFOV camera designed and built at the University of Leicester [4]. The HCGC is based on a CsI:Tl scintillator coupled to an electron multiplying CCD. The HCGC uses a pinhole collimator and an additional optical component allows for simultaneous and coaligned optical imaging [5].

An earlier iteration of the HCGC has previously been fully characterised using adapted LFOV protocols [6]. The HCGC used in this report was adapted slightly for greater sensitivity and now uses a $1500 \mu \mathrm{m}$ thick columnar CsI:Tl scintillator and a $0.5 \mathrm{~mm}$ or $1 \mathrm{~mm}$ diameter pinhole collimator. The sensitivity and FHWM spatial resolution of this system at an imaging distance of $100 \mathrm{~mm}$ are $9.8 \mathrm{~mm}$ and $0.5 \mathrm{cps} / \mathrm{MBq}$ respectively.

Each gamma photon detected by the HCGC produces a 'light splash' of scintillation photons on the CCD [4]. HCGC gamma images are reconstructed using an automatic scale space selection algorithm to fit each individual light splash [4]. Reconstructed images may then be viewed either in centre point mode - where pixel values indicate the calculated number of incoming gamma photons - or in cumulative mode - where pixel values indicate the calculated number of scintillation photons. The centre point mode is equivalent to that displayed by LFOV cameras and additional smoothing 

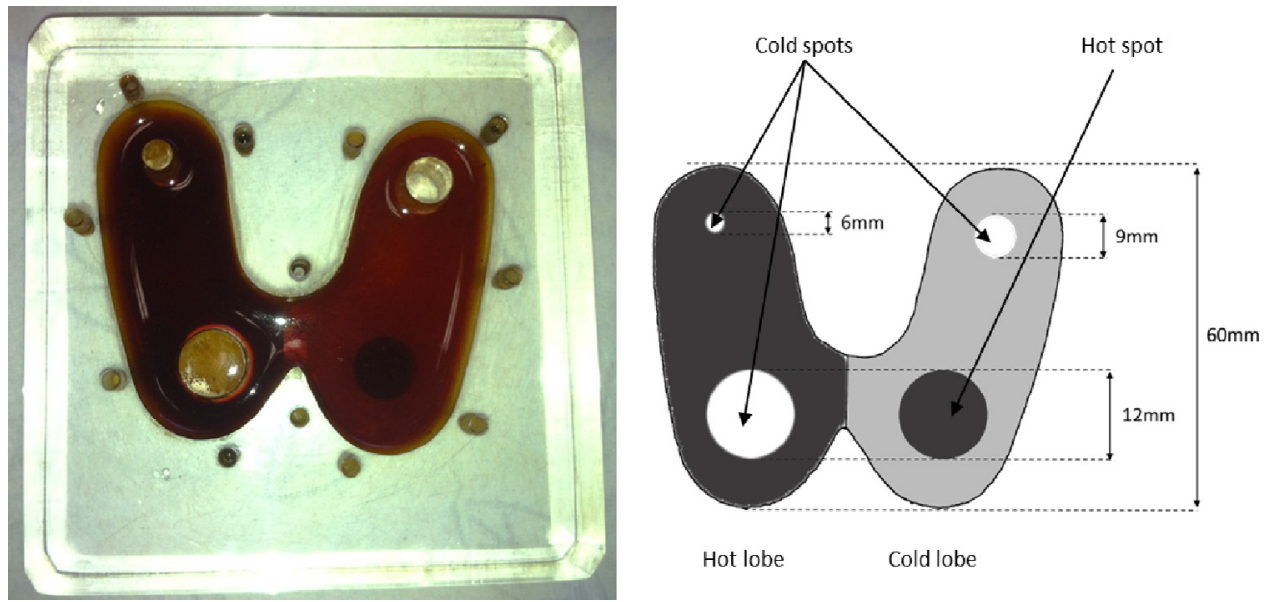

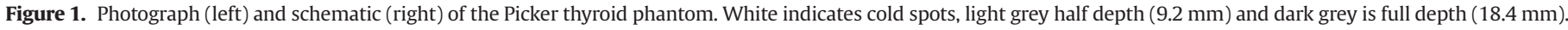

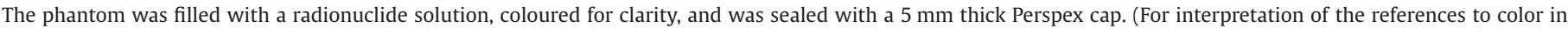
this figure legend, the reader is referred to the web version of this article.)

may be applied to improve image quality. Cumulative mode is, in effect, a smoothed version of the centre point image where the width of the smoothing filter is variable - this presentation is the most similar to the raw data recorded.

\section{Phantom specification}

The Picker (Picker Nuclear, Part \# 3602, Cleveland, OH) thyroid phantom (Fig. 1) is widely available in nuclear medicine departments and allows direct comparisons to be made with previously published studies. This phantom contains a number of structures which simulate features that may be seen in clinical images, including uneven uptake in each lobe of the thyroid and both hot (high activity) and cold (low activity) nodes.

The left 'hot' lobe of the phantom has a depth of $18.4 \mathrm{~mm}$, the right cold lobe has a depth of $9.2 \mathrm{~mm}$. Within these lobes are three cold nodes with diameters of $12 \mathrm{~mm}, 9 \mathrm{~mm}$ and $6 \mathrm{~mm}$ with no activity present. There is also a hot node, $12 \mathrm{~mm}$ in diameter, which has a depth of $18.4 \mathrm{~mm}$ in the cold lobe. The size of the entire phantom is approximately $60 \mathrm{~mm} \times 60 \mathrm{~mm}$, roughly $50 \%$ larger than a typical patient thyroid, with a fillable volume of $35 \mathrm{~mL}$. The hot lobe contains approximately $65 \%$ of the fillable volume, the hot node $1 \%$ and the cold lobe (not including the hot node) $34 \%$.

\section{Quantifying the detectability of image features}

A number of parameters can be used as measures of detectability. Contrast is defined as

$C=\frac{\left|M_{R O I}-M_{\text {background }}\right|}{M_{\text {background }}}$

where $M$ is the mean counts per pixel in the specified region [7]. These measures can be applied either using known activity in the source (subject contrast) or the counts recorded in each pixel (image contrast).

Contrast to noise ratio (CNR) is defined as

$\mathrm{CNR}=\frac{\left|M_{R O I}-M_{\text {background }}\right|}{\sigma_{\text {background }}}$

where $\sigma$ denotes a standard deviation [8]. This measure takes into account the effect of noise on detectability in addition to the contrast between ROI and background counts. To determine whether a feature is detectable, a threshold in CNR is used. Typically the CNR threshold used, as defined by Rose [9], is taken to be 3-5 [10] although this comes with a number of caveats as the Rose criterion is strongly dependent on the size of the image and the size of the lesion (or test element) being investigated. Exceeding the CNR threshold shows that the image feature is statistically likely to be real rather than due to random noise fluctuations; however it does not necessarily mean that this feature will appear visible to an operator.

\section{ROI definition}

To enable comparison with a previous study by Seret [11], ROIs were defined as follows: the ROI of interest for each node was centred on that node, with background ROIs positioned at the centre of each lobe. ROIs of the same physical size as used by Seret were achieved for the HCGC using diameters of 16,11 and 8 pixels for the background, $12 \mathrm{~mm}$ nodes, and $9 \mathrm{~mm}$ node respectively.

\section{Comparison to LFOV systems}

Since LFOV gamma cameras are regularly used in clinical thyroid imaging, a comparison between the performance of these systems and the HCGC should give an indication of the HCGC's suitability for clinical imaging. Seret [11] performed a contrast study on 52 camera heads from commercially available conventional LFOV cameras using the Picker thyroid phantom. For this study, Seret's methodology was recreated to test the HCGC.

The Picker phantom was filled with $75 \pm 5 \mathrm{MBq}{ }^{99 \mathrm{~m}} \mathrm{Tc}$ solution and imaged from $100 \mathrm{~mm}$. An image was acquired over $8 \mathrm{~min}$ - the approximate acquisition time used by Seret for each camera head. Due to the current energy resolution of the HCGC [6], an energy window of $\pm 25 \%$ was used for the HCGC data collection. A uniformity correction was applied to all images [6]. Centre point images were used in this comparison.

\section{Clinical simulation}

A typical ${ }^{99 \mathrm{~m}} \mathrm{Tc}$ thyroid scan will require administration to the patient of $185 \mathrm{MBq}-370 \mathrm{MBq}$ of activity, resulting in a typical uptake of $1 \%-5 \%$ in the thyroid [12] (note that administered activities will vary depending on location, e.g. $80 \mathrm{MBq}$ is recommended in the UK [3]). This gives an expected thyroid activity ranging from $1.85 \mathrm{MBq}$ 
a.

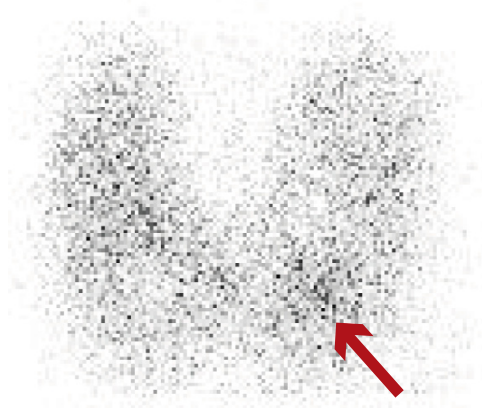

b.

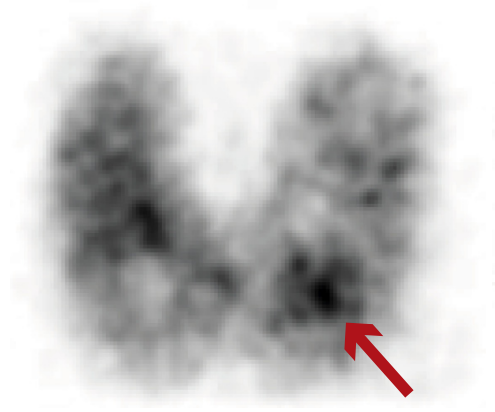

C.

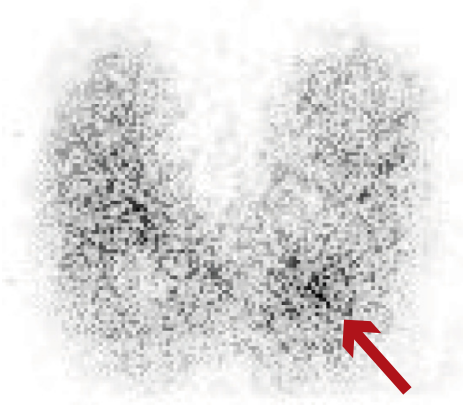

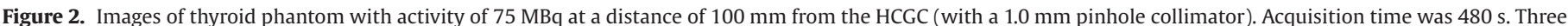

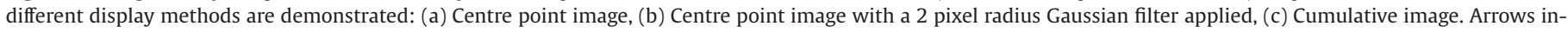
dicate approximate location of $12 \mathrm{~mm}$ hot node.

to $18.5 \mathrm{MBq}$. Planar images are acquired, with exposure times of 5 min (300 s) per view typical [12].

The thyroid phantom was filled with an activity of $15 \pm 3 \mathrm{MBq}$ ${ }^{99 \mathrm{~m}} \mathrm{Tc}$ solution and imaged over 9000 frames, resulting in a total imaging time of approximately $15 \mathrm{~min}$. The phantom-camera distance was $100 \mathrm{~mm}$. Due to the lower activity, the $1.0 \mathrm{~mm}$ pinhole collimator was used. Uniformity correction was applied prior to smoothing the images with a 2.0 pixel width Gaussian filter. Images were analysed in cumulative increments of $1 \mathrm{~min}$.

\section{Results}

Image display optimisation

Figure 2 shows a single image of the thyroid phantom displayed using three different methods. Figure 2a shows a centre point reconstructed image, Fig. $2 \mathrm{~b}$ the same centre point image with a 2 pixel radius Gaussian filter applied and Fig. 2c the cumulative reconstructed image.

Although the raw data for each image were identical, features in the processed images had different levels of detectability. This has been tabulated for the $12 \mathrm{~mm}$ hot node in Table 1. Based on the Rose threshold, this node is not clearly detectable in the centre point and cumulative images but is detectable in the smoothed image. In practice, a colour table could be applied and adjusted to provide better visibility than Fig. 2 .

\section{Effect of smoothing on image quality}

To investigate the effects of smoothing on image quality, Gaussian filters of varying widths (defined as the standard deviation of the Gaussian used) were applied to the image shown in Fig. 2a. The CNR and contrast of each node were calculated and these are compared in Fig. 3.

For a filter of width $\leq 1$, the effect on contrast was minimal. At larger filter widths, contrast was dramatically decreased. There was an improvement in CNR for all nodes up to a filter width of $\sim 2$ pixels. After this point, the rate of CNR improvement decreases and, in the case of the $12 \mathrm{~mm}$ cold node, CNR degrades when wider filters are used.

Table 1

Detectability measurements for $12 \mathrm{~mm}$ hot node for images in Fig. 2 .

\begin{tabular}{lll}
\hline Image & Contrast & CNR \\
\hline a & 1.04 & 1.01 \\
$\mathrm{~b}$ & 0.93 & 4.3 \\
$\mathrm{c}$ & 1.06 & 1.7 \\
\hline
\end{tabular}
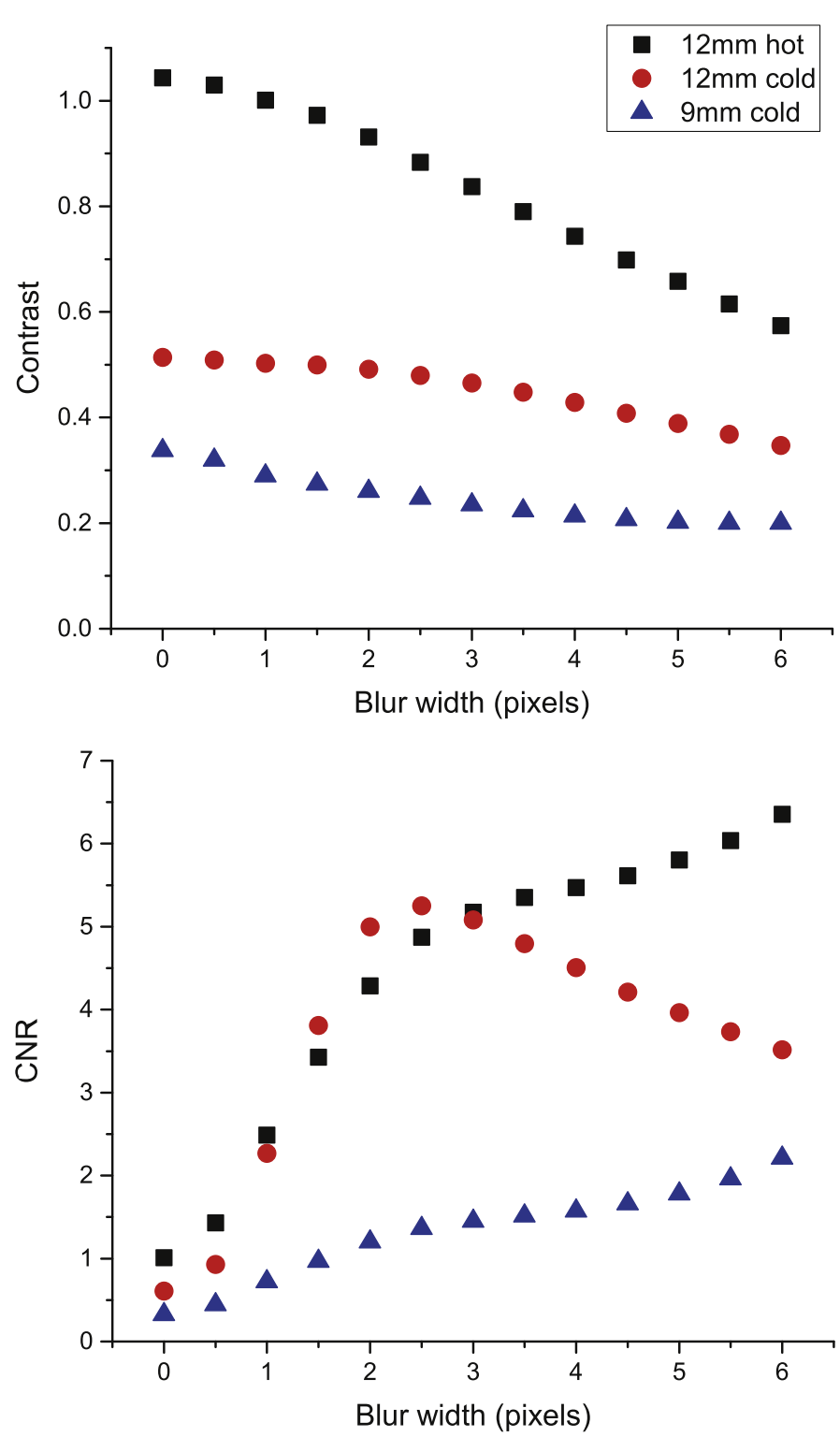

Figure 3. Contrast (top) and CNR (bottom) for each node in Fig. 2a with Gaussian filter of varying width applied. Filter width is given as the standard deviation of the applied Gaussian filter in pixels. 
Table 2

Comparison of contrast for Picker phantom from HCGC and conventional LFOV cameras under similar operating and analysis conditions. LFOV data from Seret [11]. Errors in HCGC data were derived from the standard deviation in counts within each ROI; these were large due to the low number of accumulated counts in each region.

\begin{tabular}{lllll}
\hline Area & $\begin{array}{l}\text { HCGC } \\
\text { contrast } \\
0.5 \mathrm{~mm} \varnothing\end{array}$ & $\begin{array}{l}\text { HCGC } \\
\text { contrast } \\
1.0 \mathrm{~mm} \emptyset\end{array}$ & Seret mean & Seret range \\
& $1.99 \pm 2.5$ & $1.5 \pm 0.3$ & $1.817 \pm 0.007$ & $1.739-1.873$ \\
\hline Lobe ratio & $1.47 \pm 1.5$ & $1.04 \pm 0.8$ & $0.543 \pm 0.006$ & $0.492-0.578$ \\
$12 \mathrm{~mm}$ hot node & $0.55 \pm 1.4$ & $0.51 \pm 0.5$ & $0.679 \pm 0.005$ & $0.588-0.723$ \\
$12 \mathrm{~mm}$ cold node & $0.52 \pm 1.1$ & $0.34 \pm 0.3$ & $0.429 \pm 0.005$ & $0.367-0.466$ \\
9 mm cold node & $0.5250,000$ & \\
Total image counts: & 4871 & 14,149 & 250,000 & \\
\hline
\end{tabular}

If the appropriate sized blurring filter was applied, the impact on CNR could be significant - e.g. the $12 \mathrm{~mm}$ cold spot CNR increased from 0.6 to 5.2 when a 2.5 pixel width filter was applied. This change is significant enough for the node to go from undetectable to detectable based on the Rose criterion. However there is a trade-off between contrast and CNR, with the optimum blurring width for CNR causing a decrease in contrast - particularly for small nodes.

\section{Comparison to LFOV systems}

Table 2 shows contrast values for each node in the phantom alongside the mean and range of values found in Seret's study. These take into account results from both high-resolution and ultra-highresolution collimators used by Seret, covering 30 repeats in total. The significant levels of error in the HCGC results are indicative of the poorer photon statistics compared to Seret's measurements.
Table 2 shows a general trend for enhanced contrast when the smaller diameter, higher resolution, $0.5 \mathrm{~mm}$ diameter pinhole collimator was used. However, the number of detected photons was significantly lower for this pinhole collimator and, in a low activity scenario, this effect may outweigh any improvements in contrast.

\section{Clinical simulation}

Figure 4 shows a dynamic study simulating thyroid imaging. Images are shown in $1 \mathrm{~min}$ integration time increments.

Visually, the shape of the thyroid becomes clearly defined at $\sim 2$ min and there is little change seen in the image after $\sim 8$ min.

The contrast of the $12 \mathrm{~mm}$ hot and cold nodes, and the ratio between the lobes, changed significantly over the first $3 \mathrm{~min}$ with the lobe ratio and $12 \mathrm{~mm}$ hot node contrast decreasing and the $12 \mathrm{~mm}$ cold node contrast increasing. After $5 \mathrm{~min}$, all of these contrasts had reached a level value, with a maximal deviation from the mean of $<15 \%$.

CNRs varied significantly with time, in some cases increasing or decreasing by more than $100 \%$ due to low photon statistics. The overall trend of CNRs was to increase with time.

Contrasts and CNRs calculated were lower than those seen in images taken with higher activity (e.g. those in Fig. 2), particularly for the hot node, even when integration time was restricted to keep the total imaging counts constant.

\section{First patient image}

A 49 year old male patient undergoing thyroid scintigraphy at the nuclear medicine clinic at Queen's Medical Centre, Nottingham

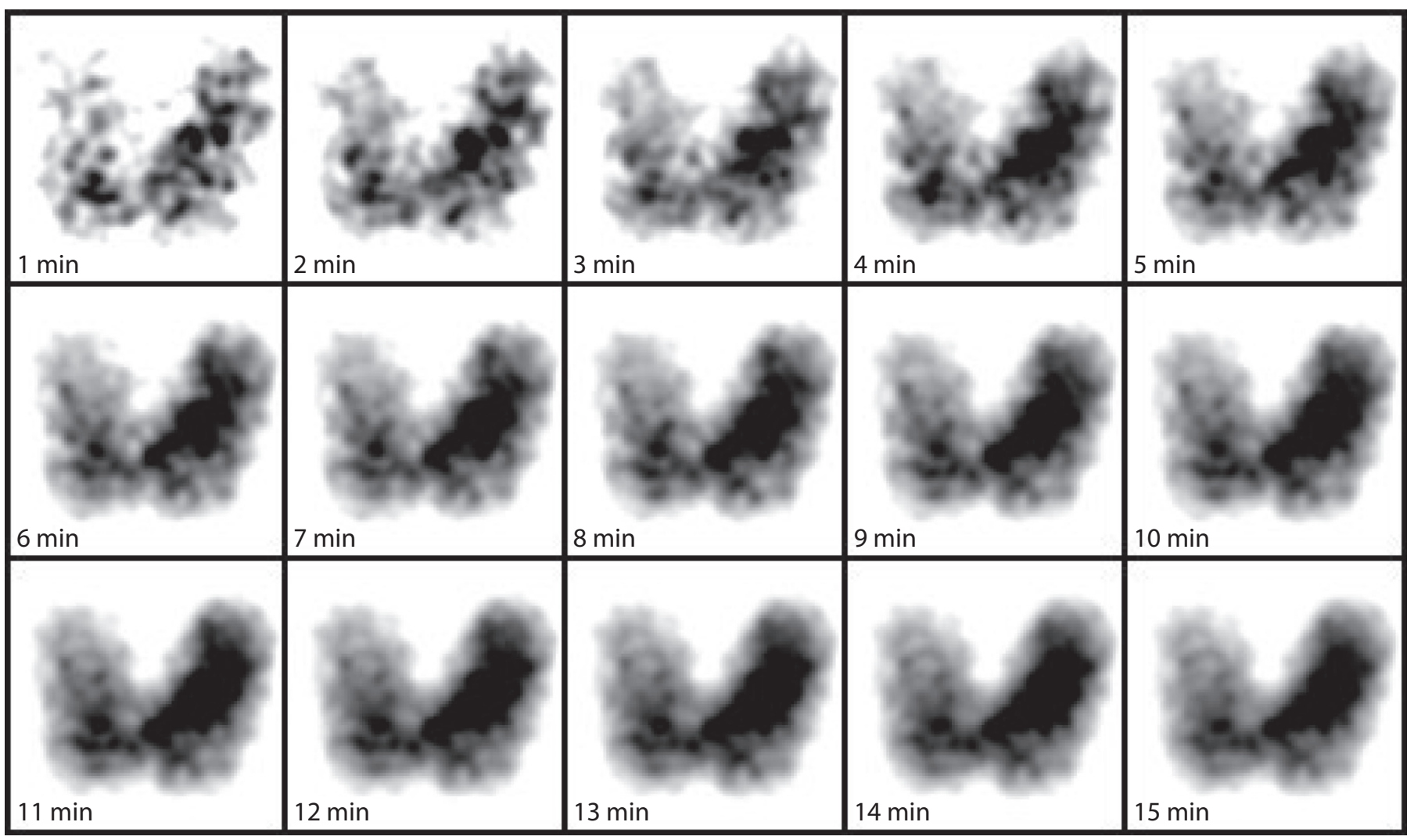

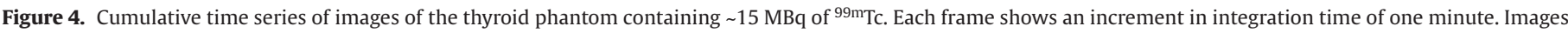
are centre point, with a 2.0 pixel Gaussian smoothing filter applied. A $1.0 \mathrm{~mm}$ diameter pinhole collimator was used. 

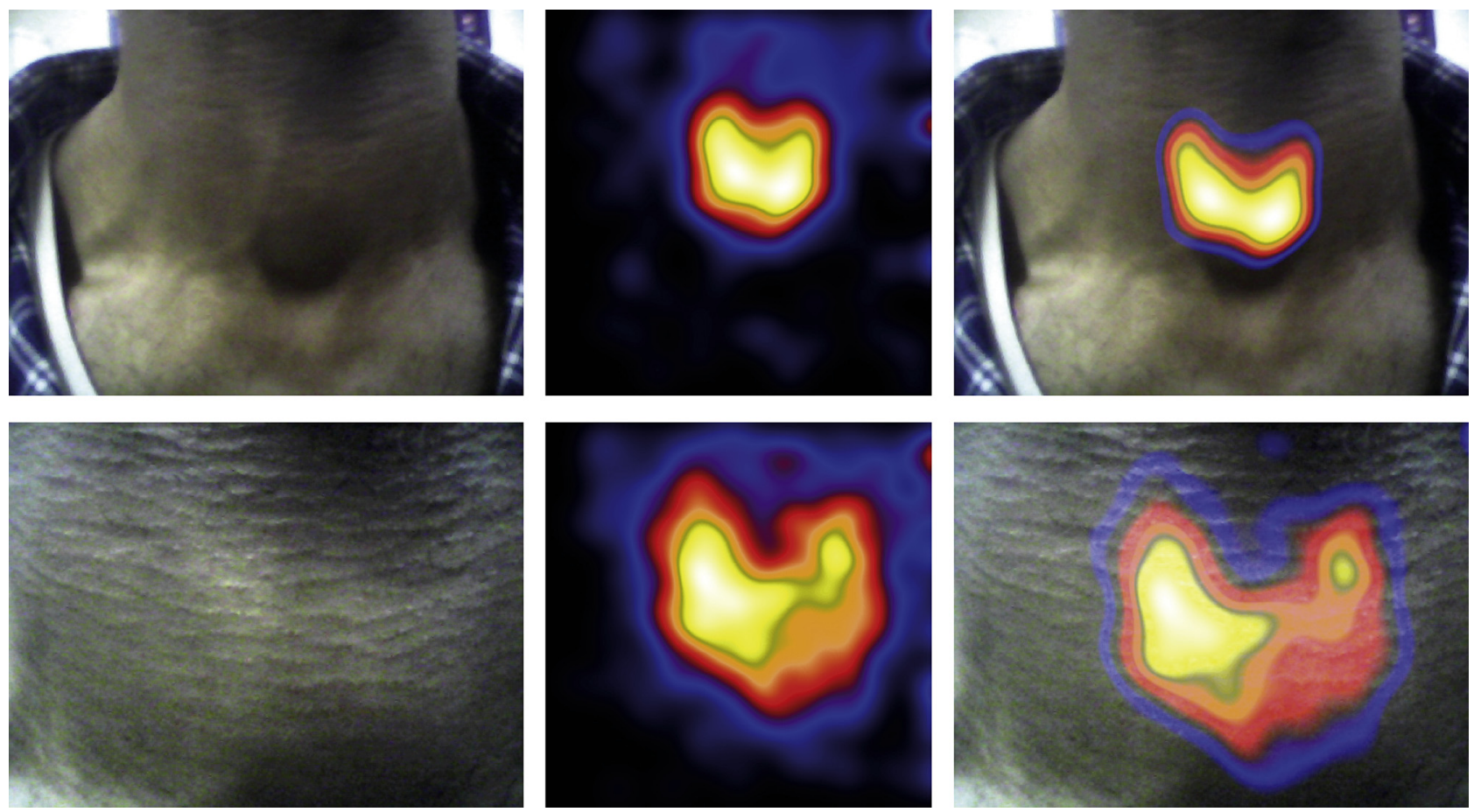

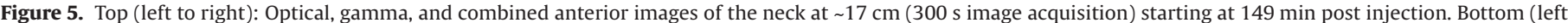

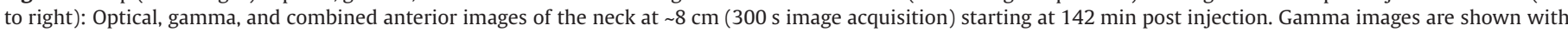
a 5 pixel width smoothing filter applied.

University Hospitals NHS Trust was imaged with the HCGC after their standard clinical test, as permitted following ethical approval.

A standard dose of $18.5 \mathrm{MBq}$ of ${ }^{123} \mathrm{I}-\mathrm{NaI}$ (159 keV) [3] was administered intravenously $140 \mathrm{~min}$ prior to patient imaging. Figure 5 shows two sets of HCGC patient images.

An initial $300 \mathrm{~s}$ image at a neck-to-camera distance of $\sim 17 \mathrm{~cm}$ was used to localise uptake (Fig. 5 top). An image was also taken with the camera closer to the patient (at a distance of $\sim 8 \mathrm{~cm}-$ Fig. 5 bottom) and a $300 \mathrm{~s}$ image was acquired.

In each set of images, the left is an optical image of the patient's neck. The gamma image (centre) is then combined with the optical image to form a hybrid image (right). The gamma image colour table has been adjusted for clarity in the hybrid image.

The thyroid images show a more active right lobe with less activity present in the left lobe (i.e. on the RHS of the images in Fig. 5). A region of higher uptake can be seen at the top of the left node. This pattern of uptake was confirmed by imaging (not shown) using a standard LFOV gamma camera. Quantification from the LFOV image indicated that $19.5 \%$ of the administered activity was taken up by the thyroid. This gave an estimation of thyroid activity of $3.2 \mathrm{MBq}$ at the time of imaging, suggesting an HCGC sensitivity of around $1.6 \mathrm{cps} / \mathrm{MBq}$ at $8 \mathrm{~cm}$.

\section{Discussion}

\section{Image display optimisation}

Both contrast and CNR were higher in the unprocessed cumulative image (Fig. 2c) compared to the unprocessed centre point image (Fig. 2a). Smoothing the centre point image had a minimal impact on contrast but significantly increased CNR due to the reduction in random noise fluctuations. Blurring in this way will however degrade the spatial resolution. Contrast in the cumulative image was similar to that of the raw image, however CNR was lower than in the blurred centre point image.
Due to the increased CNR when compared with raw images, the cumulative image will be the best form of display during camera operation. Although optimal CNR is achieved with a smoothed centre point image, the ideal level of smoothing will vary based on the image being viewed and in some cases will have a significant effect on spatial resolution and so should not be applied automatically.

\section{Effect of smoothing on image quality}

The detected size of a feature, such as a node in the thyroid phantom, will depend on its physical size and the spatial resolution of the camera system. If a blur is applied to the image then this will also act to change the apparent size of the feature.

If the node profiles are considered to be Gaussian, a reasonable assumption for large source-camera distances, the size of the image of the node may be described by the standard deviation of the fitted Gaussian $\sigma$. Any Gaussian smoothing applied can also be defined by its standard deviation. The effect of smoothing on the imaged size of the node is then expected to be $\sigma=\sqrt{\sigma_{\text {node }}^{2}+\sigma_{\text {filter }}^{2}}$, where $\sigma$ is the standard deviation of the component Gaussians.

For a filter width of 1.5 pixels - an example chosen as it showed approximately the best collective response in Fig. 3 - this would cause an expected change in imaged node size of $\sim 2 \%$ for a $12 \mathrm{di}$ ameter lesion and $\sim 7 \%$ for a $6 \mathrm{~mm}$ diameter lesion when imaged from this distance.

This increase in imaged node size due to smoothing is effectively a degradation of the spatial resolution of the system and should be taken into account when using smoothing to improve image CNR.

\section{Comparison to LFOV systems}

The $12 \mathrm{~mm}$ hot node contrast for images obtained using the HCGC exceeded the contrasts reported by Seret. When a $0.5 \mathrm{~mm}$ diameter pinhole was used, an improvement was also seen in the contrast of the $9 \mathrm{~mm}$ cold node and in the lobe ratio. 
The subject contrast for the $12 \mathrm{~mm}$ hot node was 1 (Eq. 1); this would be expected to provide an upper limit for possible measured contrast values but is exceeded by the contrasts calculated from HCGC images in Table 2. The low photon statistics, particularly in the $0.5 \mathrm{~mm}$ pinhole image where the mean counts in some regions were $<1$, result in significant errors in the calculations of contrast which may be the cause of this discrepancy.

The image contrast for the $12 \mathrm{~mm}$ cold node was lower than those recorded by Seret, even for the $0.5 \mathrm{~mm}$ pinhole where the $9 \mathrm{~mm}$ cold node contrast was higher. This is likely to be due to the less restrictive energy windowing used for HCGC results - due to lower energy resolution this was chosen to be $\pm 25 \%$, whereas LFOV cameras such as those used by Seret set windows of $\pm 10 \%$ or lower. The effect of the larger energy window would be the inclusion of more scattered counts than in an LFOV image, which would have a particularly detrimental effect on cold nodes in the high activity area of the phantom.

These results suggest that the spatial resolution achieved by the HCGC can lead to an improvement in contrast. Results further suggest that improvements in reducing background counts through energy windowing would be beneficial for thyroid imaging, along with increasing sensitivity to improve photon statistics.

\section{Clinical simulation}

These results suggest that an imaging time of approximately double that currently used clinically would be appropriate for thyroid imaging with the HCGC. This, however, will be strongly dependent on the activity uptake within the thyroid. In practice, imaging would occur in closer proximity to the thyroid when using the HCGC.

The contrast values recorded in this test were lower than those recorded for LFOV comparisons, even when acquisition time was adjusted to give very similar total counts in each image. CNRs, however, were very similar between the two sets of data. The difference between these two tests is the amount of activity present within the thyroid phantom - $75 \mathrm{MBq}$ for the LFOV comparison and $15 \mathrm{MBq}$ for the clinical simulation in Fig. 4. This suggests that background count levels are significant in the HCGC data.

\section{First patient image}

The phantom studies in this work used ${ }^{99 \mathrm{~m}} \mathrm{Tc}$, a radionuclide commonly used in thyroid imaging. In the patient image, however, emissions from ${ }^{123}$ I were imaged. This radionuclide has a primary emission energy of $159 \mathrm{keV}$ which is similar to $141 \mathrm{keV}$ for ${ }^{99 \mathrm{~m}} \mathrm{Tc}$. No previous characterisation of the HCGC had been carried out for ${ }^{123}$ I; however the images shown here confirm that the HCGC can operate using this energy.

The optical component of the HCGC was used to localise the site of uptake with a distance sufficient to image the whole neck of the patient. Once the activity is localised, the camera can brought into closer proximity to the appropriate area and an image accumulated. This technique may not be required for thyroid scintigraphy, where the expected location of activity is well defined, but may be beneficial in other procedures such as during intraoperative imaging. There was a strong agreement between the LFOV and HCGC gamma images acquired in terms of pattern of uptake.

\section{Conclusion}

From these studies, the HCGC has been shown to be suitable for clinical thyroid imaging and was comparable to LFOV systems in terms of image contrast. The detectability of image features was shown to depend on image smoothing, acquisition time and activity level. The calculated contrasts using the HCGC varied from those found for LFOV systems but these differences were smaller than the significant error on these calculations (see Table 2).

Reducing the diameter of the pinhole collimator used was shown to greatly increase image contrast, in some cases the contrast of images taken with a 0.5 diameter pinhole collimator exceeded those found with LFOV systems. The phantom tests were carried out with a camera-to-source distance of $100 \mathrm{~mm}$ to allow direct comparison to LFOV data. However, one of the practical benefits of SFOV cameras is the ability to position the detector close to the patient. At smaller distances $(<100 \mathrm{~mm})$, both the sensitivity and the spatial resolution are improved and contrast results are also expected to improve.

Feature detectability was improved with greater imaging times, however it was found that CNR and contrast were dependent on the amount of activity present, even when the number of accumulated counts was fixed. This demonstrates that it is important for phantom studies to be carried out with the appropriate activity levels. Future improvement of the energy windowing capabilities of the HCGC is also expected to improve feature detectability, particularly for low activity sources, by reducing the number of scattered photons detected.

The initial clinical study has shown that the HCGC is able to detect and image ${ }^{123} \mathrm{I}$ uptake in a patient thyroid with an acquisition time comparable to those for LFOV clinical studies.

\section{Conflict of interest}

SLB, AHN and MSA declare that they have no conflicts of interest. JEL and ACP are directors of Gamma Technologies Limited, a spin out company of the Universities of Leicester and Nottingham.

\section{Acknowledgements}

The authors would like to thank Elaine Blackshaw, Nottingham University Hospitals NHS Trust, for assistance in collecting the results used in this report, Oliver Blake, University of Leicester, for software development, and David Monk, Helen Hill, and Chris Hastings, Leicester Royal Infirmary, for providing ${ }^{99 \mathrm{~m}} \mathrm{Tc}$ for this work. This work was supported by an STFC CLASP grant (ST/I00327/4). SLB is supported by a Science and Technology Facilities Council studentship (ST/J501104) and AHN is sponsored by the Ministry of Health, Malaysia.

\section{Appendix Supplementary material}

Supplementary data to this article can be found online at doi:10.1016/j.ejmp.2015.12.002.

\section{References}

[1] Tsuchimochi M, Hayama K. Intraoperative gamma cameras for radioguided surgery: technical characteristics, performance parameters, and clinical applications. Phys Med 2013;29(2):126-38.

[2] Bhatia BS, Bugby SL, Lees JE, Perkins AC. A scheme for assessing the performance characteristics of small field-of-view gamma cameras. Phys Med 2015; 31(1):98-103.

[3] British Nuclear Medicine Society. BNMS clinical guidelines: radionuclide thyroid scans, <http://www.bnms.org.uk/procedures-guidelines/bnms-clinical -guidelines/radionuclide-thyroid-scans.html>; 2003 [accessed 02.15].

[4] Lees JE, Bassford DJ, Blake OE, Blackshaw PE, Perkins AC. A high resolution Small Field Of View (SFOV) gamma camera: a columnar scintillator coated CCD imager for medical applications. J Instrum 2011;6(12):C12033.

[5] Lees JE, Bugby SL, Bark AP, Bassford DJ, Blackshaw PE, Perkins AC. A hybrid camera for locating sources of gamma radiation in the environment. J Instrum 2013;8(10):P10021.

[6] Bugby SL, Lees JE, Bhatia BS, Perkins AC. Characterisation of a high resolution small field of view portable gamma camera. Phys Med 2014;30(3):331-9.

[7] Seret A. A Comparison of Contrast and Sensitivity in Tc-99m Thyroid Scintigraphy between Nine Nuclear Medicine Centres of a geographic area. Alasbimn J 2006;8(32):AJ32-3. 
[8] Dickerscheid D, Lavalyle J, Romijn L, Habraken J. Contrast-noise-ratio (CNR) analysis and optimisation of breast-specific gamma imaging (BSGI) acquisition protocols. EJNMMI Res 2013;3:21.

[9] Rose A. The visual process. vision: optical physics and engineering. Springer US; 1974

[10] Cherry SR, Sorenson JA, Phelps ME. Physics in nuclear medicine. 3rd ed. PA, USA: Elsevier; 2003.
[11] Seret A. Hot and cold contrasts in high-resolution Tc-99m planar scintigraphy: a survey of fifty-two camera heads using the PICKER thyroid phantom. Phys Med 2010;26(3):166-72.

[12] Mettler FA Jr, Guiberteau MJ. Essentials of nuclear medicine imaging. Elsevier Health Sciences; 2011 